\title{
Présence de Grillons du genre Myrmecophilus à l'île de la Réunion (Orthoptera, Myrmecophilinae) \\ Sylvain Hugel, Fabrice Blard
}

\begin{abstract}
First sighting of Myrmecophilus crickets on Réunion island (Orthoptera, Myrmecophilinae).

Two ant-loving crickets are observed for the first time on Réunion island (Indian Ocean), Myrmecophilus quadrispinus Perkins, 1 899, and M. americanus Saussure, 1877 . This is the first observation of the former outside the Pacific area. Distinctive characters between M. americanus and M. cottami Chopard, 1922, are discussed as well as the synonyms of Myrmecophilus microscopicus Gorochov, 1994. The synonymy between M. microscopicus and M. albicintus Chopard, 1924, is removed.
\end{abstract}

\section{Résumé}

Deux espèces de Grillons myrmécophiles sont mentionnées de la Réunion : Myrmecophilus quadrispinus Perkins, 1899, et $M$. americanus Saussure, 1877. II s'agit de la première observation du premier hors de la région Pacifique. Les caractères distinctifs de $M$. americanus et $M$. cottami Chopard, 1922, sont discutés ainsi que les synonymes de Myrmecophilus microscopicus Gorochov, 1994. La synonymie entre M. microscopicas et M. albicinctus Chopard, 1924, est annulée.

\section{Citer ce document / Cite this document :}

Hugel Sylvain, Blard Fabrice. Présence de Grillons du genre Myrmecophilus à l'île de la Réunion (Orthoptera, Myrmecophilinae). In: Bulletin de la Société entomologique de France, volume 110 (4-5), octobre/décembre 2005. pp. 387389 ;

https://www.persee.fr/doc/bsef_0037-928x_2005_num_110_4_16251

\section{Ressources associées :}

Myrmecophilus

Orthoptera

Fichier pdf généré le 04/10/2019 


\title{
Présence de Grillons du genre Myrmecophilus à l'île de la Réunion (Orthoptera, Myrmecophilinae)
}

\author{
par Sylvain HUGEL ${ }^{*} \&$ Fabrice BLARD ${ }^{* *}$ \\ "Institut de Neurosciences cell. et integr., LC2, UMR 7168 CNRS, 21 rue Descartes, F - 61084 Strasbourg cedex ch \\ $<$ hugel@neurochem.u-strasbg.fr> \\ *Insectarium de la Réunion, F-97420 Le Port <fabrice.blard@wanadoo.fr>
}

\begin{abstract}
Résumé. - Deux espèces de Grillons myrmécophiles sont mentionnées de la Réunion: Myrmecophilus quadrispinus Perkins, 1899, et $M$. americanus Saussure, 1877. Il s'agit de la première observation du premier hors de la région Pacifique. Les caractères distinctifs de $M$. americanus et $M$. cottami Chopard, 1922, sont discutés ainsi que les synonymes de Myrmecophilus microscopicus Gorochov, 1994. La synonymie entre $M$. microscopicus et $M$. albicinctus Chopard, 1924, est annulée.

Summary. - First sighting of Myrmecophilus crickets on Réunion island (Orthoptera, Myrmecophilinae). Two ant-loving crickets are observed for the first time on Réunion island (Indian Ocean), Myrmecophilus quadrispinus Perkins, 1899, and $M$. americamus Saussure, 1877. This is the first observation of the former outside the Pacific area. Distinctive characters between $M$. americanus and $M$. cottami Chopard, 1922, are discussed as well as the synonyms of Myrmecophilus microscopicus Gorochov, 1994. The synonymy between $M$. microscopicus and $M$. albicintus Chopard, 1924, is removed.
\end{abstract}

Key words. - Orthoptera, Myrmecophilidae, Myrmecophilinae, new geographical record, ant-loving species, Mascarene, Indian Ocean, island of Réunion.

Des îles des Mascareignes, seule l'île Maurice était connue pour abriter des Grillons appartenant au genre Myrmecophilus. Dans son article traitant de la faune orthoptérologique de l'île Maurice, VINSON (1968) mentionne la présencc d'« une petite Myrmecophila cavernicole apparemment inféodée aux grottes à hirondelles ». Cette donnée curieuse n'a pas été confirmée depuis. A la Réunion aucune espèce appartenant au genre Myrmecophilus n'était connue, mais en réalisant l'inventaire des fourmis de l'île (BLARD et al., 2003), nous avons trouvé des grillons de ce genre associés à plusieurs espèces de fourmis. Deux espèces bien distinctes ont été trouvées.

Myrmecophilus quadrispinus Perkins, 1899. - Les spécimens prélevés partagent avec cette espèce l'ensemble des caractères externes et internes disponibles (voir DESUTTER-GRANDCOLAS, 1997). Des mâles et des femelles de cette espèce ont été trouvés, dans ou près de nids de fourmis Paratrechina longicornis (Latreille, 1802) et Solenopsis geminata (Fabricius, 1804), suggérant une reproduction sexuée de cette espèce à la Réunion. Les spécimens ont été prélevés sur la commune du Port (altitude $<10 \mathrm{~m}$ ) de janvier à mars 2003 et en février 2004.

$M$. quadrispinus a été cité de milieux anthropisés à Honolulu, Hawaii, où il est considéré comme une espèce importée (HEBARD, 1926) et où il était déjà connu pour parasiter des fourmis de ces genres (ZIMMERMAN, 1948; OTTE, 1994). TRAVIS, 1941, indique que Solenopsis geminata abrite des Myrmecophilus indéterminés en Floride et Géorgie. M. quadrispinus a été par la suite retrouvé dans d'autres îles de l'océan Pacifique: les Samoa (CHOPARD, 1929), les îles de la Loyauté en Nouvelle-Calédonie (DESUTTER-GRANDCOLAS, 1997) et à Hong-Kong. Ainsi, l'observation de $M$. quadrispinus à la Réunion est la première mention de cette espèce hors de la région Pacifique.

Myrmecophilus americanus Saussure, 1877. - Les spécimens prélevés partagent avec cette espèce la coloration, l'armure des tibias et métatarses postérieurs, l'oviscapte aux valves en 
forme de cuillère et la forme des palpes, tels qu'illustrés par SCHIMMER (1909) et comme chez les syntypes (Museum für Naturkunde der Humboldt-Universität, Berlin). Nous avons trouvé quatre femelles de cette espèce dans ou près de nids de $P$. longicornis, Le Port, XII.2003.

Comme $M$. quadrispinus, $M$. americanus est connu pour parasiter les fourmis du genre Paratrechina (WASMANN, 1905; CHOPARD, 1963) ; d'après ce dernier, $M$. americanus est «très répandue dans les pays à climat chaud »" Ce grillon a été signalé de Colombie (SAUSSURE, 1877), du Brésil (en tant que M. prenolepidis ; WASMANN, 1905), d'Hispaniola (PEREZ-GELABERT, 2001), d'Hawaii (HEBARD, 1926; OTTE, 1994), d'Israël (CHOPARD, 1963'), de l'est de la Libye (CAPRA, $1929^{1}$ ), de l'est de l'Inde (en tant que M. prenolepidis ${ }^{1}$; WASMANN, 1905) et peut-être des Seychelles ( $c f$. remarques ci-dessous).

Les deux espèces de Myrmecophilus que nous avons trouvées à la Réunion parasitent des espèces pantropicales de fourmis sans doute introduites. La localisation de ces grillons semble restreinte aux milieux anthropisés de basse altitude, suggérant que ces Myrmecophilus aient été introduits à la Réunion, sans doute avec leur hôte. Il se pourrait d'ailleurs que la présence commune de ces deux Myrmecophilus à Hawaii et la Réunion, îles tropicales situées aux antipodes l'une de l'autre, ne soit pas simplement anecdotique. La faune et la flore de la biosphère, et particulièrement des milieux insulaires, ont tendance à s'uniformiser sous la pression des introductions d'espèces exotiques associées aux activités humaines (LÖVEI, 1997). M. quadrispinus, $M$. americanus et leurs hôtes entrent peut-être dans la composition d'un de ces écosystèmes "mcdonaldisé" (LÖVEI, 1997), se substituant progressivement à l'environnement d'origine des îles de la planète.

\section{Remarques relatives à Myrmecophilus americanus, $M$. cottami et $M$. prenolepidis, M. microscopicus et $M$. albicinctus}

Myrmecophilus prenolepidis Wasmann, 1905, a été considéré comme synonyme de $M$. americanus par SCHIMMER (1909). Cette synonymie est souvent omise dans la littérature et pourrait être remise en cause (PEREZ-GELABERT, 2001).

Dans une note consécutive à sa description de $M$. cottami Chopard, 1922, CHOPARD (1923) précise ne pas disposer de caractère distinctif entre $M$. cottami et $M$. americanus, conduisant CAPRA (1929) à les mettre en synonymie suivant les conseils épistolaires de Chopard. OTTE (1994) considère invraisemblable que l'espèce soit identique en Colombie et en Egypte et réfute la synonymie.

Il existe pourtant un caractère distinctif entre les deux espèces : le basitarse postérieur de $M$. cottami est armé d'une unique épine au milieu (CHOPARD, 1922), alors que SCHIMMER (1909) illustre le basitarse postérieur de $M$. americanus armé de deux épines : une proximale et une distale. Une seule épine très proximale est encore décelable sur les basitarses postérieurs des syntypes très abimés de $M$. americanus (la partie distale du tarse est encollée ou couverte de débris rendant impossible l'observation d'une éventuelle épine distale), ce qui contraste avec la localisation médiane de l'unique épine de $M$. cottami. Sur les quatre $M$. americanus prélevés à la Réunion, un spécimen nè présentait qu'une seule épine sur les basitarses postérieurs. La localisation de cette épine était très proximale comme celle de la première épine des spécimens en comportant deux. Une variation intraspécifique du nombre d'épines du basitarse postérieur existe donc chez $M$. americanus comme cela semble par exemple être le cas chez $M$. hirticaudus Fischer-Waldheim, 1846 (HARTZ, 1969), mais dans le cas où une seule épine est présente, sa localisation n'est pas médiane.

M. microscopicus Gorochov, 1994 et M. albicinctus Chopard, 1924. - M. microscopicus dont seul le mâle est décrit a été placé en synonymie sans justification avec $M$. albicinctus dont seule la femelle est décrite (ICHIKAWA, 2001). Si la coloration et l'armure des tibias postérieurs semblent identiques chez ces deux espèces, $M$. microscopicus est près de deux fois plus petit que M. albicinctus (1,8 mm contre 3,5 mm). Un tel dimorphisme sexuel n'a jamais été documenté dans cette sous-famille, et l'auteur de cette synonymie décrit d'ailleurs une nouvelle espèce de

\footnotetext{
${ }^{1}$ Peut-être s'agissait-il de M. cottami ?
} 
Myrmecophilus sur un unique critère de taille (ICHIKAWA, 2001). Outre la taille, l'armure des basitarses postérieurs est différente chez ces deux espèces, et la variabilité de ce caractère n'est pas documentée pour ces deux taxons. La synonymie entre $M$. microscopicus et $M$. albicinctus nous paraît donc injustifiée dans l'état actuel des choses.

En revanche, il ne peut être exclu que $M$. microscopicus décrit des Seychelles d'après un unique mâle ne soit un synonyme de $M$. americanus dont seule la femelle est décrite. En effet, la petite taille $(\approx 2 \mathrm{~mm}$ pour $M$. americanus $)$, la couleur, la forme des palpes et des fémurs postérieurs et l'armure des tibias et basitarses postérieurs pourraient correspondre d'autant que nous venons de découvrir $M$. americanus dans la même région. Seul le prélèvement de spécimens de $M$. americanus des deux sexes provenant de la même colonie de fourmis pourra permettre d'éclaircir ce point de manière satisfaisante.

REMERCIEMENTS. - Nous remercions la Société entomologique de France pour la bourse Germaine Cousin attribuée à S. H. en 2002, Laure Desutter-Grandcolas pour ses remarques constructives et son soutien, et Michael Ohl du Museum für Naturkunde der Humboldt-Universität de Berlin pour la mise à disposition de photographies détaillées des syntypes de $M$. americanus.

\section{AUTEURS CITÉS}

BlaRd F., DoROW W.H.O. \& DelaBie J.H.C., 2003. - Les Fourmis de l'île de la Réunion (Hymenoptera, Formicidae). Bulletin de la Société entomologique de France, 108 (2): 127-137.

CAPRA F., 1929. - Risultati zoologici della Missione inviata dalla R. Società Geografica Italiana per l'esplorazione dell'oasi di Giarabub. Ortotteri e dermatteri. Annali del Museo Civico di Storia Naturale "Giacomo Doria", 53 : 122-159.

ChOPARD L., 1922. - Description d'un nouveau Myrmecophila (Orth. Gryllidae) du Soudan égyptien. Bulletin de la Société entomologique de France, $27: 42-44$.

1923. - Note sur quelques fórmes du genre Myrmecophila (Orth. Gryllidae) et diagnose d'une sous-espèce nouvelle de France. Bulletin de la Société entomologique de France, 28 : 29-30.

1963. - Notes sur la faune des Orthoptéroïdes d'Israel et de quelques régions limitrophes (Blattodea, Gryllacridoidea, Gryllodea). Bulletin of the Research Council of Israel, 10 B : 161-176.

— 1968. - Orthopterorum Catalogus. Pars 12, Fam. Gryllidae: subfam. Mogoplistinae, Myrmecophilinae, Scleropterinae, Cachoplistinae, Pteroplistinae, Pentacentrinae, Phalangopsinae, Trigonidiinae, Eneopterinae; Fam. Oecanthidae, Gryllotalpidae. M. Beier's gravenhage, Dr W. Jung N. V.: 215-500.

DESUTTER-GRANDCOLAS L., 1997. - First record of ant-loving crickets (Orthoptera, Myrmecophilidae, Myrmecophilinae) in New Caledonia. Journal of the Australian Entomological Society, 36 : 159-163.

HARZ K., 1969. - Die Orthopteren Europas 1. Series entomologica 5 : 749 p. The Hague (Dr W. Junk N.V.).

HEBARD M., 1926. - Records of Hawaiian Dermaptera and Orthoptera of the family Gryllidae. Proceedings Hawaiian Entomological Society, 6:292-303.

LöVEI G. L., 1997. - Global change through invasion. Nature, $338:$ 627-628.

OTTE D., 1994. - The crickets of Hawaii: origin, systematics and evolution. Orthopterists' Society, Philadelphia, 1-396 p.

- 1994. - Orthoptera Species, File 1. Academy of natural Sciences of Philadelphia.

Perez-GelaberT D. E., 2001. - Preliminary checklist of the Orthoptera (Saltatoria) of Hispaniola. Journal of Orthoptera Research, 10 (1): 63-74.

PERKINS R.C.L., 1899. - Orthoptera - Fauna hawaiiensis. In: Sharp D. (Ed.), 2 (1) : 1-30 ; Cambridge (Cambridge University Press).

SCHIMMER F., 1909. - Beitrag zu einer Monographie der Gryllodeengattung Myrmecophila Latr. Zeitschrift für Wissenschaftliche Zoologie, 93 : 409-534.

TRAVIS B.V., 1941. - Notes on the biology of the fire-ant Solenopsis geminata (F.) in Florida and Georgia. Florida Entomologist, $24: 15-22$.

VINSON J., 1968. - Les Blattoptéroïdes, Orthoptéroïdes et Dermaptéroïdes des Mascareignes. The Mauritius Institute Bulletin, 6 (3): 103-115.

WASMANN E., 1905. - Zur Lebensweise einiger in- und ausländischer Ameisengäste. Zeitschrift für Wissenschaftliche Insekten-Biologie, 1 : 329-336.

ZiMMERMAN E.C., 1948. - Insects of Hawaii. Vol. 2. Apterygota to Thysanoptera. Honolulu (University of Hawaii Press), $475 \mathrm{p}$. 